\title{
Power Spectrum Analysis of EEG Signals for Estimating Visual Attention
}

\author{
Mitul Kumar Ahirwal \\ National Institute of Technology Raipur,
}

Raipur-492010

\author{
Narendra D londhe \\ Assistant Professor \\ National Institute of Technology Raipur, \\ Raipur-492010
}

\begin{abstract}
The task oriented brain activity analysis and classification is a prime issue in EEG signal processing these days. The similar attempt has been done here to estimate the brain activity on the basis of power spectrum analysis. For this, the modified approach involving both Independent Component Analysis (ICA) and Principal Component Analysis (PCA) methodologies has been used in this paper to investigate the behavior of brain's electrical activity for a simple case of visual attention. The proposed method of EEG classification can be very useful in predicting the action or the intention of action performed on the basis of EEG which leads to more development in brain computer interface. The EEG data has been referred from a website and the mathematical tool for EEG analysis called EEGLAB has been used to perform work in this paper.
\end{abstract}

\section{General Terms}

Visual attention, Signal processing, Estimation.

\section{Keywords}

EEG, ICA, PCA, Power spectrum.

\section{INTRODUCTION}

It is well known fact that the EEG signals are the measure of the vigilance state of brain which changes according to task performed by a person. These changes are classified into few different frequency bands named as delta, theta, alpha, beta and gamma [1-2]. The accurate classification of electrical activity for a particular state of human brain helps in neurological diagnosis and also for establishing standards for instrumentation development. This classification also helps in the brain computer interfacing [3-4] which has been gaining wide attraction in the research industry. On this line of research, several studies have been conducted like correlation of awake and sleep states of brain [5], estimation of visual and audio alertness [6]. Task related to alertness of human controller of ship, airplane, truck, rail are analyzed with the help of EEG signals. For this, mathematical transform and artificial neural network techniques have been used. The power spectrum analysis has been also used in the research mentioned earlier but in complex form and also found dependent upon error rate variation in time. Power estimation and changes in EEG signals related to muscle fatigue over the motor cortex area for Adductor Pollicis muscle measured in Relaxed and contraction states are classified using change in power spectrum [7], studies like Test of Variables of Attention (TOVA) was performed with power estimation and PCA to measure alertness[8]. Feature extraction also done with the help of power spectral entropy in case to recognize left and right hand movement [9]. To evaluate the human stress power spectrum analysis is done in some studies which results in related frequencies identification, IQ indexing with the help of EEG signals in terms of correlation between different frequency band is also calculate by power spectrum analysis [10-11].In this paper, the EEG signals of visual attention task have been analyzed. For this EEG data set of 32 channels [12] has been used. Firstly, the signal has been filtered to remove unwanted noise from the signal. In the second stage of signal processing, the artifacts have been removed by trimming signal manually throughput the length of signal. After the preprocessing of EEG signals, Independent Component Analysis (ICA) has been performed on signals from 32 channels to separate out various components. Next, in order to perform single stage analysis, all the components have been converted into a single component by using Principal Component Analysis (PCA). The principle component has been decomposed in different frequency classes of EEG signal i.e. delta, theta, alpha, beta frequency bands and power spectrum analysis has been done for each.

\section{METHODOLOGY}

The following steps are involved in this work shown in the Figure. 1. Starting from loading the EEG signals and filter out the unwanted frequency after that artifact are removed. Initial power spectrum of all channels are generated after that Independent component analysis and principal component analysis are applied to significantly notice the power spectrum of each frequency band, these steps are performed in EEGLab[13] .

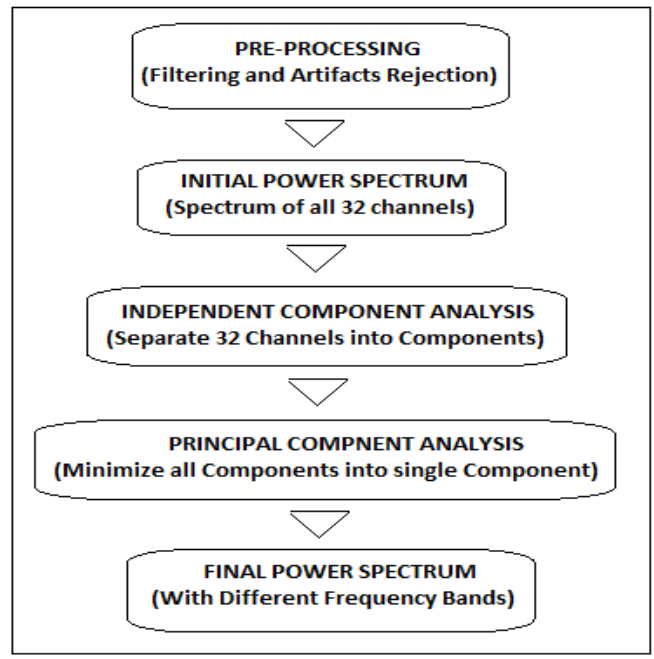

Fig.1. Flow graph of various steps involved in work 


\subsection{EEG SIGNAL PREPROCESSING}

The EEG signal filtering of recorded EEG signals has been done in the range of frequencies $0.1 \mathrm{~Hz}$ to $60 \mathrm{~Hz}$ by applying FIR filter of second order. At the same time, notch filter is also used to remove the power line interference in the channels spectrum around $50 \mathrm{~Hz}$. The artifacts are the disturbances in all or few channels due to eye blink or sometime by the low conductivity of electrodes. These disturbances are easily visible in time domain representation of the signals. These artifacts are present in all the channels, so that portion of data has been trimmed off manually from the signals all channels at once.

\subsection{INITIAL POWER SPECTRUM ANALYSIS}

Secondly, initial power spectrum of the brain activity has been observed. Figure. 2 shows the signal power distribution along the range of frequencies. From Figure, it can be easily observed that power spectrums of all channels are closely concentrated and overlapping. On analyzing these graphs, it is really difficult to comment on the power levels delta, theta, alpha, beta frequency bands. The high power levels can be easily observed in delta and beta frequency classes.

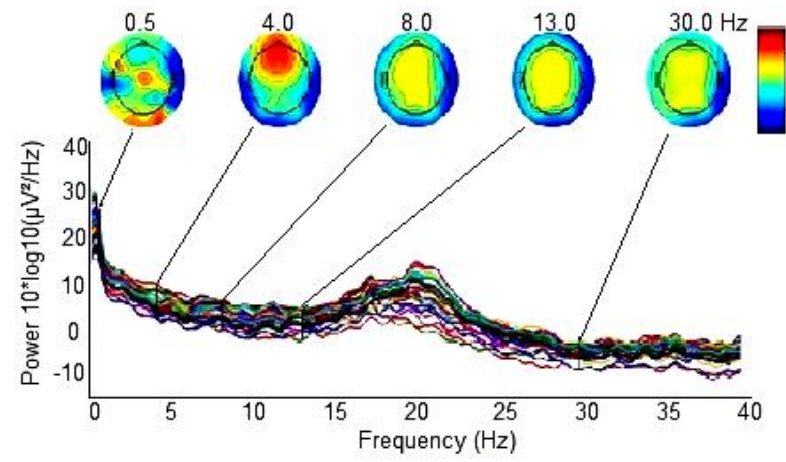

Fig.2. Activity power spectrums of all channels before PCA

To see the variations significantly, the signals are processed with Independent Component Analysis (ICA) to separate out into individual components.

\subsection{INDEPENDENT COMPONENT ANALYSIS (ICA)}

The EEG signals are generally recorded through multichannel lead system. Some important mutual information existing between pairs of channels is greatly valuable to reveal brain state information, build models, and establish locations etc. The most commonly used method for EEG processing are Fourier analysis and wavelet transform. These methods are appropriate for single channel EEG data because of its strong randomness and non-stationary nature. But these methods are not suitable for predicting the inter-dependence of the data of different channels in case of multichannel data processing. Most recently proposed the ICA algorithm is a statistical signal processing method, which can effectively separate the statistically independent source signals from observed multichannel signals, also used in improving the detection of changes in EEG [14-15]. ICA can be simply described as follows: assuming that there are $N$ number of observed signals $X_{i}$, where $i=1, \ldots, N$, from $N$ number of electrodes, and each signal is a linear mixture of $N$ independent source signals $S_{i}$.

$$
X=A S
$$

Where $X=\left[x_{1}, \ldots, x_{N}\right]^{T}$ is observed signal vector and $S=\left[s_{1}, \ldots\right.$, $S_{N} l^{T}$ is source signal vector, $A$ is an unknown $N x N$ mixing matrix. We expect that the source signal $S$ can be separated from the observed signal vector $\boldsymbol{X}$. However, as the independent source $\boldsymbol{S}$ and mixing matrix $\boldsymbol{A}$ both are unknown, the traditional signal processing methods cannot solve the problem of blind source separation (BSS). Up to now, a lot of theories and algorithms for ICA have been presented and used to estimate the separation matrix $\boldsymbol{W}$ so that the independent sources can be successfully separated from observed multichannel signals, namely

$$
S=W X
$$

Independent Component Analysis (ICA) decomposes the input data using either logistic infomax ICA algorithm with the natural gradient feature or optionally the extended-ICA algorithm [16] with sign estimation $N$ training blocks. If $N=0$, automatically estimate the number of sub-Gaussian sources, use single training block $(N=1)$. The extended ICA has been followed here for input data decomposition.

\subsection{PRINCIPAL COMPONENT ANALYSIS (PCA)}

The PCA is a classical multivariate data analysis method that is useful in linear feature extraction, data compression and dimension reduction, sometimes used for back ground noise reduction [17]. It is very much equivalent to KarhunenLoCve transformation and closely related to factor analysis. These all methods follow the second order statistical computation. The PCA finds a linear transformation $V=W_{u}$ such that the retained variance is maximized. It can be also viewed as a linear transformation which minimizes the reconstruction error. The row vectors of $W$ correspond to the normalized orthogonal eigenvectors of the data covariance matrix. One simple approach to PCA is to use singular value decomposition (SVD) [18-19]. Let us denote the data covariance matrix by $R_{u}=E\left\{u u^{\prime}\right\}$. Then the SVD of $R_{u}$ gives

$$
\mathrm{R}_{\mathrm{u}}=\mathrm{U}_{\mathrm{u}} \mathrm{D}_{\mathrm{u}} \mathrm{U}_{\mathrm{u}}^{\prime}
$$

Where $U_{u}$ is the eigenvector matrix (i.e., modal matrix) and $D_{u}$ is the diagonal matrix whose diagonal elements correspond to the eigen values of $R_{u}$. Then the linear transformation $\mathrm{W}$ for PCA is dominant given by $W=U_{u}^{\prime}$.For dimensionality reduction, one can choose $\mathrm{p}$ column vectors in $U_{u}$ that are eigenvectors that have the largest eigen dimensions. Annealing based on weight changes is used to automate the separation process. 32 components are now reduced to single value to construct a linear transform $W$. Conversion of all components into single component is done with PCA to reduce component. This single component gives more accurate reading of Activity Power Spectrum corresponding to different frequencies. Figure. 3 show the single component activity power spectrum.

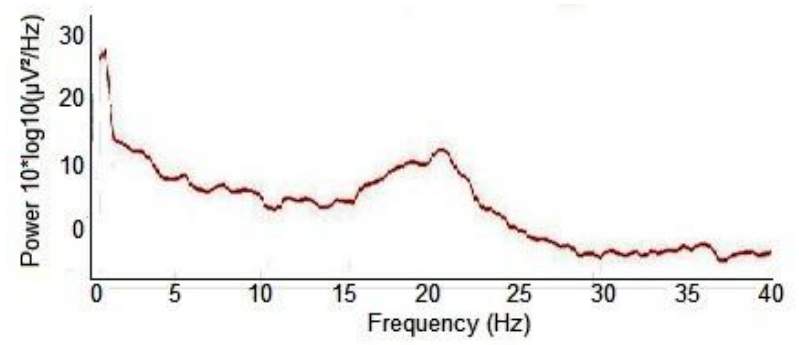

Fig.3. Activity Power Spectrum of Single Component after PCA 


\subsection{SEPARATION OF FREQUENCY BANDS AND FINAL POWER SPECTRUM ANALYSIS}

The separation of different frequency classes $(0.1-4 \mathrm{~Hz})$ delta, $(4-8 \mathrm{~Hz})$ theta, $(8-13 \mathrm{~Hz})$ alpha and $(13-30 \mathrm{~Hz})$ beta for EEG signals are performed here. For this, use the FIR digital bandpass filters. Now, compute the power spectrum of each to analyze the different frequency bands. The low frequency and high amplitude EEG signals are generated at the time of sleep or in relaxed state of healthy person, while the high frequency and lower amplitude EEG signals are generated in awake and working state of person.

\section{RESULTS}

In this study, the EEG data of visual attention task of one subject (80 trials) (32-channel).Electrodes are placed according to the 10-20 system and total time of recording is $119 \mathrm{sec}$, signals are sampled at frequency of $256 \mathrm{~Hz}$. Above specified data after processing gives following results shows in Figure. 3 and Table 1.

From Figure.3, it can be observed that the maximum signal power is up to $30 \mathrm{~dB}$. Normally, freq range from 0.1-4(Delta) and 4-8(theta) are generated in sleep and relaxed condition. Our EEG signals contains some of these components and shows maximum power in range of $0-5 \mathrm{~Hz}$ but same power levels also reaches in frequency bands of $15-25 \mathrm{~Hz}$ and rest of frequencies have low power levels. From Figure.4, the following observations are found.

Categorized readings of each of frequency band is given below and also arranged in Table 1 .

- $0.1-4$ (delta) - in this range, the maximum power is $25-30 \mathrm{~dB}$ corresponding to $0-0.5 \mathrm{~Hz}$

- $4-8$ (theta) - in this range, the maximum power is $8-9 \mathrm{~dB}$ corresponding to $4.5-5 \mathrm{~Hz}$

- $8-13$ (alpha) - in this range, the maximum power is $6-7 \mathrm{~dB}$ corresponding to $8.5-9 \mathrm{~Hz}$

- 13-30(beta) - in this range, the maximum power is $10-12 \mathrm{~dB}$ corresponding to $18-22 \mathrm{~Hz}$

Table 1. Frequency bands and power variation table

\begin{tabular}{|c|r|r|r|c|}
\hline $\begin{array}{c}\text { Frequency } \\
\text { Band }\end{array}$ & $\begin{array}{c}\text { Max. } \\
\text { Power } \\
\text { (dB) }\end{array}$ & $\begin{array}{c}\text { Min. } \\
\text { Power } \\
\text { (dB) }\end{array}$ & $\begin{array}{c}\text { Max. } \\
\text { Power } \\
\text { Freq } \\
\text { (Hz) }\end{array}$ & $\begin{array}{c}\text { Min } \\
\text { Power } \\
\text { Freq } \\
\text { (Hz) }\end{array}$ \\
\hline $0-4$ & $25-30$ & $10-11$ & $0-0.5$ & $3.5-4$ \\
\hline $4-8$ & $8-9$ & $6-6.5$ & $4.5-5$ & $6-6.5$ \\
\hline $8-13$ & $6-7$ & $3.5-4$ & $8.5-9$ & $10-11$ \\
\hline $13-30$ & $10-12$ & $0-1$ & $18-22$ & $28-30$ \\
\hline
\end{tabular}

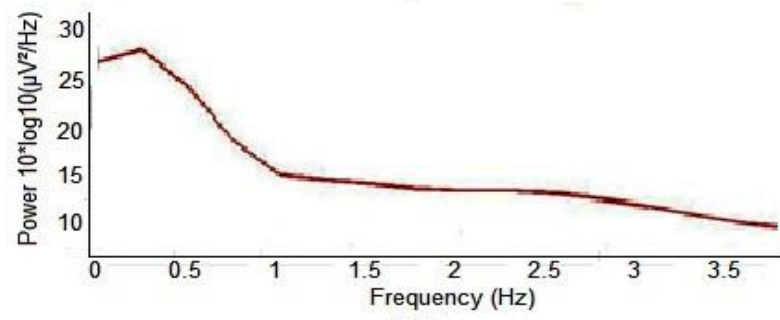

(A)

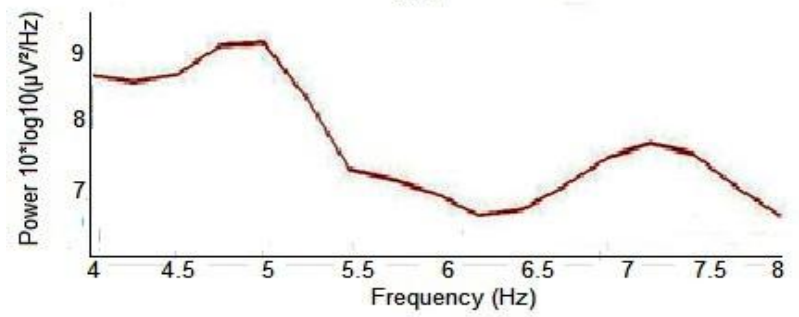

(B)

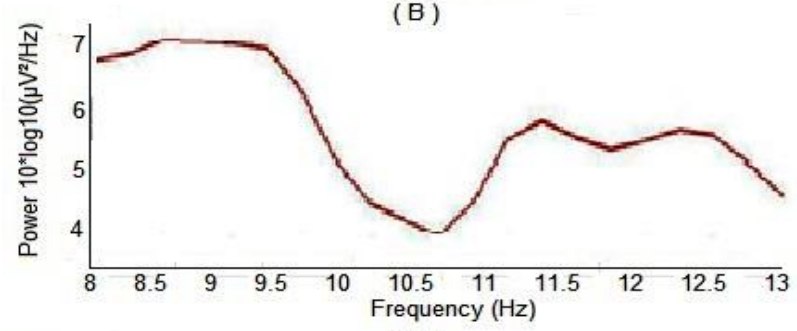

(C)

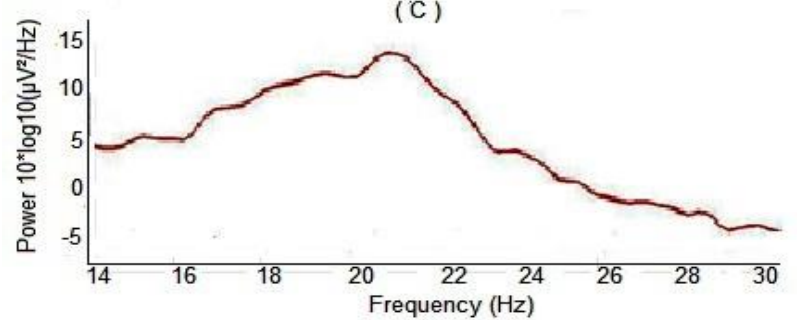

(D)

Fig.3 Power spectrums of (A)delta(0.1-4 Hz) frequency band, (B) theta $(4-8 \mathrm{~Hz})$ frequency band, $(\mathrm{C})$ alpha $(8-13 \mathrm{~Hz})$ frequency band and $(D)$ beta $(13-30 \mathrm{~Hz})$ frequency band

\section{CONCLUSION}

From this study of power spectrum we concluded that the power in signals while performing visual attention task is near $10-12 \mathrm{~dB}$ having frequency range of $18-22 \mathrm{~Hz}$. visual attention frequencies lies in the $(13-30 \mathrm{~Hz})$ beta band of EEG signals classification, this frequency band is related to alertness or awakens of human beings .Above results shows that inside the beta band of EEG signals, frequency range of $18-22 \mathrm{~Hz}$ having power of $10-12 \mathrm{~dB}$ is generated corresponding to cognitive process of brain or visual attention .This study is helpful for designing a system in clinical researches that was able to check the defects or problem in cognitive task of brain by matching the patient EEG signals with above results sets . Normally, persons who are in the professions that needs to perform visual attention task more than normal person may be victim of defects in visual or cognitive tasks after a certain period of time. 


\section{ACKNOWLEDGMENTS}

Our thanks to the Mr.Arnaud delorme for providing public database for EEG data with proper information of data format and instruction regarding use of data.

\section{REFERENCES}

[1] Sanei, S., and Chambers, J.A., EEG signal processing, John Wiley \& Sons Ltd. 2007.

[2] Pfurtschellera, G., Lopes, F.H., Silva, Da., "Event-related EEG/MEG synchronization and desynchronization: basic principles", Clinical Neurophysiology, 1999.

[3] Schalk, G., McFarland, D.J., Hinterberger, T., Birbaumer, N., Wolpaw, J.R., "BCI2000: a general-purpose brain-computer interface (BCI) system," IEEE Transactions on Biomedical Engineering, 2004

[4] Fabiani, G., McFarland, D.J., Wolpaw, J.R., and Pfurtscheller, G., "Conversion of eeg activity into cursor movement by braincomputer interface (bci)", IEEE Trans. on Neural Systems and Rehabilitation Eng., 2004

[5] Kiymik, K.M., Akin, M., and Subasi, A., "Automatic recognition of alertness level by using wavelet transform and artificial neural network", Journal of Neuroscience Methods, 2004.

[6] Jung, T.P., Makeig, S., Stensmo, M., Sejnowski, T.J., "Estimating Alertness from EEG power spectrum", IEEE Transaction on Biomedical Engineering, 1997.

[7] Abdul-latif, A.A., Cosic, I., Kumar, D.K., Polus, B., and Costa, C.Da. 2004., Power changes of EEG signals associated with muscle fatigue: the root mean square analysis of EEG bands, Intelligent Sensors, Sensor Networks and Information Conference.

[8] Luzheng, Bi., Zhang, R., Zhilong, C. 2007 Study on Real-time Detection of Alertness Based on EEG, IEEE/ICME International Conference on Complex Medical Engineering.

[9] Zhang, A., Yang, B. and Huang, L. 2008 Feature Extraction of EEG Signals Using Power Spectral Entropy, Paper Presented at the BioMedical Engineering and Informatics Conference BMEI.

[10] Hamid N.H.A., Sulaiman, N., Aris, S.M.A., Murat Z.H., and Taib, M.N., 2010 Evaluation of Human Stress Using EEG
Power Spectrum, Paper Presented at the International Colloquium on Signal Processing \& Its Applications (CSPA).

[11] Lias, S., Sulaiman, N., Murat, Z.M., and Taib, M.N. 2010 IQ Index using Alpha-Beta Correlation of EEG Power Spectrum Density (PSD), Paper Presented at the IEEE Symposium on Industrial Electronics and Applications (ISIEA) Penang, Malaysia.

[12] EEG/ERP data vailable for free public download. Obtained through the Internet: http: //sccn.ucsd.edu / arno/fam2data publicly_available_EEG_data.html, [accessed 15/01/2011].

[13] EEGLAB a Matlab tool box for EEG analysis Obtained through the Internet:http://sccn.ucsd.edu [accessed 12/11/2010].

[14] Makeig, S., Bell, A.J., Sejnowski, T.J., and Jung, T.P. "Independent Component Analysis of Electroencephalographic Data", Advances in Neural Information Processing Systems. MIT Press, Cambridge MA, PP.145-151, 1996.

[15] Van Dun, B.; Wouters, J.; Moonen, M., "Improving Auditory Steady-State Response Detection Using Independen Component Analysis on Multichannel EEG Data," IEEE Transactions on Biomedical Engineering, vol.54, no.7, pp.12201230, July 2007

[16] Jung, T.P., Humphries, C., Lee, T.W., Makeig, S., McKeown, M.J., Iragui, V., and Sejnowski, T.J., "Extended ICA removes artifacts from electroencephalographic recordings", Advances in Neural Information Processing Systems , MIT Press, Cambridge, 1998

[17] Jolliffe, I.T., Principal Component Analysis, 2nd Edition. Spinger, 2002.

[18] Lei, C., Jie L., Yaoru S., Zhu, H., Yan, C. 2010 EEG-based vigilance analysis by using fisher score and PCA algorithm, IEEE International Conference on Progress in Informatics and Computing (PIC).

[19] Oh, C., Kim, M.S., and Lee, J.J. 2006, EEG signal classification based on PCA and NN, Paper Presented at the SICE-ICASE International Joint Conference, Korea. 